\title{
Editorial: Nationalismus am Ende des 20. Jahrhunderts
}

I.

Nicht weniger als das Ende des osteuropäischen Sozialismus überrascht die Intensität der allerorts aufbrechenden nationalistischen Konflikte. In Europa, dem Ausgangsort der 'Universalgeschichte', galt die mit dem Zeitalter der Französischen Revolution eingeleitete Konstitution von Nationalstaaten spätestens seit dem 2. Weltkrieg als abgeschlossen. Ein blutiger Prozeß, immerhin aber schien nach 1945 Gewalt als Mittel zur Veränderung staatlicher Grenzen für alle Zeiten diskreditiert.

"Unser Vaterland heißt Europa*, lautete die Reaktion vieler Deutscher auf die nationalsozialistische Katastrophe. Die aus der Eskalation militårischer Gewalt und aus dem deutschen Rassenwahn gezogene Lehre bestand darin, die Gefahr künftiger Kriege durch die politische Einigung Europas zu entschärfen - auch wenn daraus, nach Beginn des Ost-West-Konflikts, zunächst nur die westeuropäische Integration wurde. Zwar galt in allen westlichen Ländern das *nationale Interesse* nach wie vor als höchster Wert staatlicher Politik. Nationalistische Bewegungen im engeren Sinn wurden jedoch eher als Gefahr für die parlamentarische Demokratie eingeschåtzt. Immerhin hatte die westdeutsche Politik, auch wenn dazu zweieinhalb Jahrzehnte erforderlich waren, im Zuge der Ostpolitik gelernt, die bestehenden Grenzen aller Staaten in Europa, das hieß insbesondere auch die Polens, als unverletzlich anzuerkennen. Die westliche Linke dachte ohnehin in universalistischen Kategorien und sympathisierte allenfalls mit den "nationalen Befreiungsbewegungen in der Dritten Welt.

Allerdings war der Spielraum für nationalistische Sonderwege, für Abspaltungen und neutralistische Bestrebungen angesichts der übergeordneten Konkurrenz der Machtblöcke eng begrenzt. Aber nicht allein die Einbindung in militårische Bündnisse, auch der greifbare Vorteil frei zirkulierender Waren und eines freizügigen Reiseverkehrs zumindest innerhalb Westeuropas half, nationale Ressentiments weitgehend abzubauen. Und so schien die im 19. Jahrhundert aufgebaute Konkurrenz zwischen den Prinzipien des Nationalismus, des Sozialismus und der Demokratie in den westlichen Nachkriegsgesellschaften zu einem gewissen Ausgleich gebracht. Nach außen in das Netz internationaler Institutionen eingebunden, sicherte die weiterhin geltende nationalstaatliche Ordnung ihren Bürgern Rechtssicherheit und und ein Mindestmaß an sozialer Gerechtigkeit. Und auch im Osten Europas waren Bürgerkriege und die Rivalitäten ethnischer Gruppen (ein sehr fragwürdiger Terminus) unter dem Druck der Bündnis- 
systeme bis auf weiteres stillgestellt.

Mit dem Schwinden der sowjetischen Hegemonie brechen nun überall überwunden geglaubte Konflikte hervor: eine explosive Kombination von wechselseitigen Gebietsansprïchen, Flüchtlingsströmen und wheimzuholenden* Minderheiten - ein bitterer Abgesang auf den »proletarischen Internationalismus*. Spätestens seit dem Spanischen Bürgerkrieg sollte freilich klar geworden sein, daß die 1919 gegründete III. Kommunistische Internationale nie mehr als eine Außenstelle der KPdSU und ein Instrument zur Ausschaltung oppositioneller Strömungen im Ausland gewesen war. $\mathrm{Zu}$ keiner Zeit hatte sich der sowjetische Internationalismus von strategischen Motiven getrennt. Das Machtkalkůl, das bei allen Abkommen der Sowjetunion mit den osteuropäischen Ländem im Vordergrund stand, hat jeden Versuch einer überstaatlichen Zusammenarbeit im Keim vergiftet. Breschnews Doktrin der »begrenzten Souveränität sozialistischer Länder*, eine nachgeschobene Rechtfertigung für die Niederschlagung des Prager Frühlings, hat hier zumindest Klarheit geschaffen. Bei den Bruderländern sollten keine weiteren Mißverständnisse über die Spielräume für wirtschafts- und außenpolitische Alleingänge aufkommen. Andererseits hat der $*$ Rat für gegenseitige Wirtschaftshilfe* nie zu einer der Europäischen Gemeinschaft vergleichbaren Integration der osteuropäischen Volkswirtschaften geführt. Herrschaftsansprüche seitens des großen Bruders, Mißtrauen seitens der 1945 hinzueroberten Länder und ein bilateralistischer Handel haben die Herausbildung supranationaler Institutionen verhindert. Der strategische Umgang mit der sogenannten Nationalitätenfrage hat sich gerächt, und auch hier zeigt sich, daß die sowjetischen Führer der eigenen Ideologie erlegen sind. *Wenn das Nationalitätenproblem nicht grundsätzlich gelöst worden wäre, hätte die Sowjetunion nicht das gesellschaftliche, kulturelle, wirtschaftliche und defensive Potential, über das sie verfügt. * Es fällt leicht, diese von Gorbatschow noch 1987 abgegebene Einschätzung von ihrem Nachsatz her zu widerlegen. Offenbar konnte weder das mit enormen ökologischen Kosten erkaufte Wachstum noch die staatlich verordnete Einheitskultur gesellschaftliche Loyalität gewährleisten, so daß die Gesellschaften »sowjetischen Typs* ohne ständige Pråsenz der staatlichen Macht nicht regierbar gewesen wären. Nach der Erschöpfung des sowjetischen Modells scheint es nur natürlich, daß die politische Initiative an nationalistische Bewegungen übergegangen ist ( $\mathrm{s}$. hierzu den Beitrag von Ernest Gellner). Dadurch wurden jedoch überall im ehemals kommunistischen Bereich zugleich die heißen Themen des alten Nationalismus aktualisiert: die Angst vor »Überfremdung ", vor separatistischer Zerstückelung (s. den Aufsatz von Erhard Stölting) und das Interesse an »Grenzkorrektur«。 
Die von Gorbatschow geöffnete Klammer des Ost-West-Konflikts hat freilich auch Rückwirkungen auf den westlichen Teil Europas. Sie hat den Regionalismen und Autonomiebestrebungen in den westlichen Ländern (Norditalien, Schottland, Baskenland) neuen Bewegungsraum geschaffen. $O b$ daraus separatistische Bewegungen mit Erfolgsaussichten werden, mag dahin gestellt bleiben. Beunruhigender ist die jetzt auch in den sog. Wohlstandsinseln Belgien, Dånemark und Schweden zu beobachtende Konjunktur nationalistischer Bewegungen. Die vielleicht größte Gefahr für die »europåische Friedensordnung* resultiert daraus, daß zumindest zwei westliche Bündnispartner unmittelbar in die heißen Nationalismen Süd-Osteuropas involiert sind. In Griechenland werden einerseits die eigenen Landsleute aus dem nördlichen Nachbarstaat als Wirtschaftsflüchtlinge abgeschoben; andererseits kursieren bereits Karten, auf denen Griechenland bis in die Nähe Tiranas reicht. Für die Türkei gelten die gegen die alte Sowjetunion so erfolgreich ins Spiel gebrachten Grundsätze der Autonomie und des Minderheitenschutzes offenbar nicht. Die Kurden können in dem von türkischer Seite gegen sie geführten Krieg kaum auf westliche Unterstützung hoffen, da in dieser Region nach wie vor strategische Interessen, wenn nicht mehr gegenüber dem Kommunismus, so jetzt gegenüber dem islamischen Fundamentalismus überwiegen. Eine neue Situation wird allerdings dann eintreten, wenn die Türkei meint, ihren jüngst entdeckten »historischen Verpflichtungen * gegenüber der Integrität Aserbaidschans mit militärischen Mitteln nachkommen zu müssen. Unabhångig von diesen Entwicklungen hat „Europa* als politischer Bezugspunkt eine folgenreiche Wandlung durchlaufen. „Europa* fungiert nicht mehr allein als eine Idee* der Integration, sondern auch als ein Rangschema, das allzu durchsichtigen Interessen den Schein einer kulturhistorischen Ableitung verleiht. So soll die relative Nähe zu Europa Anwartschaften auf eine bevorzugende Hilfe sichern - etwa gemäß der feinsinnigen Unterscheidung zwischen dem »europafreundlichen Nationalismus* der Kroaten und dem »parakommunistischen Nationalismus* Serbiens (vgl. hierzu den Aufsatz von Nicole Janigro). Andererseirs sind erste Umrisse eines europäischen Nationalismus sichtbar geworden, der in erster Linie auf Abgrenzung nach außen zielt. Die Konstruktion gemeinsamer Sitten und einer europäischen Kultur dient dazu, eine europäische Außengrenze zur Abwehr unkontrollierter Zuwanderung und eine Mauer gegen »den Islam* zu legitimieren - auch wenn dadurch die historischen Bindungen etwa Spaniens zu Lateinamerika, die Năhe Frankreichs und Italiens zum Magreb verloren gehen und für eine beträchtliche Anzahl eingewanderter Europäer diskriminierende Hindernisse im Verkehr mit ihren außereuropäischen Verwandten entstehen. 
II.

Wie viele Ereignisse der jüngeren Zeit widersprechen die nationalistischen Regungen allen theoretischen Erwartungen: "Idealismus, deutsche Geschichtsphilosophie, Liberalismus, Sozialdarwinismus und moderne Soziologie haben an dieser Klippe ebenso Schiffbruch erlitten wie der Marxismus*, schrieb Tom Nairn lange vor der großen Welle des jüngsten Nationalismus, in der die letzten Versuche, den $*$ realen Sozialismus* zu reformieren, untergegangen sind. Betroffen ist von dieser Entwicklung zuerst der Marxismus, da sein Internationalismus zugleich Theorie und politische Programmatik sein sollte. Kaum geringer ist die Verlegenheit der akademischen Soziologie, die ihren Gesellschaftsbegriff an der Normalität der westlichen Nachkriegsgesellschaften ausgerichtet hatte (s. hierzu den Beitrag von Heinrich Haferkamp) und auf die neue Lage mit definitorischen Anpassungsmanövern reagiert. (Einen Überblick über neuere historisch vergleichende Erklärungansätze gibt Niels Kadritzke.)

Die Rede von der *Rückkehr* des Nationalismus ist denn auch nicht zuletzt Ausdruck einer Verlegenheit, da sie die Besonderheit dieser Erscheinungen mit historischen Analogien verdeckt, und dem nationalen Mythos (s. dazu die Thesen Ludi Lodovicos), der sich selbst nur allzugern als *Wiedergeburt» präsentiert, stärker verhaftet bleibt, als ihr lieb sein kann (s. hierzu die Kontroverse zwischen Ernest Gellner und Branka Magas). Die neuen Nationalismen resultieren nicht aus verspäteten Übergangskrisen agrarischer in industrielle Gesellschaften, sie reagieren auf spezifisch $*$ modeme* Problemkomplexe. Die Sowjetunion war nicht das 'letzte Imperium', sondern zerbrach letzlich an einem Umverteilungsmechanismus zwischen den Republiken, als dieser nicht länger Wachstum, sondern die Übergangskosten zur Markłwirtschaft aufteilen sollte. Im neuen westeuropäischen Nationalismus rächt sich, daß die Herstellung des gemeinsamen Marktes ohne ausreichende sozialpolitische Absicherung verläuft und damit die Risiken auf jene Gruppen abgewälzt werden, die ihnen am wenigsten gewachsen sind. Die rassistische Phantasien freisetzende Einwanderung ist nicht zuletzt Folge unerfüllter Entwicklungsversprechen an die Länder der Dritten Welt.

Auch wenn die Vielfalt der neuen Nationalismen vorschnelle Verallgemeinerungen verbietet, wäre Eric Hobsbawm doch darin zuzustimmen, daß sie allesamt nicht in der Lage sind, die Probleme, denen sie ihren Antrieb verdanken, zu lösen. Diese Einsicht wäre freilich erst dann ein Anlaß zur Hoffnung, wenn sie zu den Akteuren durchdringt. $O b$ das geschieht, ist zur Zeit sehr ungewiß. 